\title{
The failing health care system for adult patients with cystic fibrosis
}

\author{
S P Conway, D E Stableforth, A K Webb
}

Excellent paediatric care has ensured that most children with cystic fibrosis survive into adulthood. ${ }^{12}$ Centre based care from a multidisciplinary cystic fibrosis team has been shown to deliver a better prognosis ${ }^{3}$ and continuing optimal supervision delivered from multidisciplinary adult cystic fibrosis centres has seen patient survival reach beyond the fourth decade of life. Antenatal screening, selective or universal, will eventually reduce the proportion of paediatric patients further. ${ }^{4}$ Cystic fibrosis is becoming predominantly an adult disease. The adult cystic fibrosis clinics in Birmingham, Leeds, and Manchester currently look after over 600 patients whereas five years ago this combined figure was approximately 400 . Each of the three clinics has an average annual net increase of 20 patients.

Medical management has increased survival, not only because care delivered by cystic fibrosis centres has improved with experience but also because there are more treatment options to maintain patients in a good and stable clinical state. These include intensive use of oral and intravenous antibiotics, maximised nutrition with nasogastric or gastrostomy feeding, and the prescription of novel drugs such as DNase which can potentially increase survival further. Consequently many people with cystic fibrosis have a better quality of life, often with jobs, families, and concrete hopes for the future. Much can be done for sicker patients too, helping them towards transplantation by attention to every detail of care, nasal intermittent positive pressure ventilation, and continuous intravenous antibiotics. Cystic fibrosis has become very much the province of the adult respiratory physician, and as it becomes increasingly complex so it becomes increasingly expensive to resource.

As paediatricians have transferred more and healthier patients, the adult physician has had the privilege of caring for a growing number of knowledgeable, organised, and articulate adults. However, as a consequence of this steady growth and the high expectations of patients and their relatives, the demands on some centres will outstrip their ability to continue to provide a first class service. This will show as reduced consultant input per patient, overcrowded clinics, no comprehensive annual review, reduced ward supervision, a lack of the clinical research necessary to maintain centre credibility, and poor morale amongst overworked staff. These consequences are inevitable unless the hospital trusts recognise that resources flowing to the major cystic fibrosis centres must reflect patient needs.

For some years there has been awareness of the potential medical and financial problems posed by the rapid increase in the adult cystic fibrosis population. Efforts have been made to define, recommend, and answer these needs. In 1990 the Department of Health commissioned a Clinical Standards Advisory Group ${ }^{5}$ to evaluate the access and availability of specialist cystic fibrosis services in the UK. Clinical guidelines and standards of care for adult cystic fibrosis patients have been published by the Royal College of Physicians. ${ }^{67}$ The cost of resourcing cystic fibrosis care has been carefully evaluated on several occasions. ${ }^{8-10}$ These recommendations are not being met, laying bare a lack of advance planning or central strategy development for this important group and exposing our patients to the vagaries of local initiatives and gross inequality of service between regions.

The current guidelines from the Royal College of Physicians recommend 3-4 consultant sessions and 2-3 sessions from another senior doctor per week per 50 patients. ${ }^{7}$ In the adult cystic fibrosis units at Birmingham, Leeds, and Manchester one consultant currently has overall responsibility for over 200 patients. Historically the care for adult patients with cystic fibrosis has been assumed largely by interested physicians in addition to their existing clinical workload, and some continue busy respiratory and general medical practices. As total patient numbers have increased, individuals inevitably receive less personal attention, despite the need for constant input from consultants and other experienced senior doctors, thus undermining the confidence of patients in the quality of care delivered.

The accepted level of junior doctor support for every 50 patients is five sessions of one registrar grade with a special interest in cystic fibrosis, in addition to the usual junior staffing of a medical team. The recommended professional support is: one clinical nurse specialist, two physiotherapists, one secretary, one part-time dietitian, and one part-time social worker. Based on these criteria, the levels of junior doctor and paramedical support in some of the larger regional adult cystic fibrosis units are sufficient for 100 patients, approximately half the numbers actually attending.

Many cystic fibrosis units are currently primed with monies from the UK Cystic Fibrosis Trust which enables the appointment of research fellows, cystic fibrosis clinical nurse specialists, and other necessary staff. Only some of these positions have been financed by hospital trusts. Even the inadequate infrastructure of the existing care system for adult patients with cystic fibrosis remains dependent on the continuing generosity of the Cystic Fibrosis Trust's clinical support and improvement grants, a maximum of $£ 25000$ per annum and given according to the clinic's patient numbers. These grants are often "topped" up by the cystic fibrosis unit from other charity sources and "slush funds" in order to fund full-time junior doctors. Cystic Fibrosis Trust support remains a "sine qua non" in most units and if it were withdrawn some would simply not be able to deliver effective health care.

The lack of proper support for cystic fibrosis care typifies the short sightedness of present funding levels. Medication accounts for over $60 \%$ of the total cost of cystic fibrosis care. Suboptimal care results in sicker patients who inevitably cost more. It is not inevitable, however, that older patients need vastly more expensive treatments as, with optimal care, the annual decline in pulmonary function can be marginal, complications can be dealt with promptly, and their deleterious effects minimised. Overall costs for adults could be significantly reduced if staffing levels were sufficient to allow more frequent review of patients by senior doctors and paramedical staff with special expertise in cystic fibrosis.

Adult cystic fibrosis units struggle with inadequate bed bases. Despite the enthusiastic uptake of home intravenous 
antibiotic treatment which now accounts for over half of all "iv days" in some centres, there is daily pressure on a limited number of hospital beds. There has been no advance planning for the predicted increase in adult patient numbers. The published guidelines on bed numbers, based on paediatric cystic fibrosis care, recommend six per 50 patients, but are not being met and are inadequate for an adult population with more advanced disease. Birmingham has no dedicated adult cystic fibrosis beds. For patients with Pseudomonas aeruginosa infection Leeds has eight beds and Manchester 10. Patients chronically infected with Burkholderia cepacia have to compete for beds with noncystic fibrosis patients on other acute wards.

Adult beds are increasingly blocked by severely ill patients without the domestic support to leave hospital, often for months. Emergency admissions are to wards ill equipped for the special needs of sick young adults. Acute admissions are usually accommodated within 2-3 days in Manchester, but may wait for up to two weeks in Birmingham and Leeds. Early intervention in acute respiratory exacerbations is crucial to minimise permanent lung damage. ${ }^{11}$ Patients who are failing to improve on home intravenous antibiotic treatment need admission, not merely another week of home treatment in the hope that "things will get better". Transition from the paediatric to the adult care system should be smooth and planned, but is not helped by having to mix with older adults in a general chest ward as in Birmingham. ${ }^{12}$ None of these is achievable without adequate bed numbers, staffing and dedicated beds.

The care of children with cystic fibrosis is based on regional centres and satellite care. The latter, smaller units in district general hospitals (DGH) may send their patients to the centre for annual assessments and periodically hold combined outpatient clinics in the satellite hospital. Routine admissions are to the satellite hospital. This system does not always work for adult cystic fibrosis care. Paediatric cystic fibrosis patients are usually referred directly to an adult cystic fibrosis centre and DGH respiratory physicians who, unlike their paediatric counterparts, have minimal experience of the requirements of cystic fibrosis adult care. The complex medical diversity of adult patients with cystic fibrosis demands an emphasis on centre care, probably two per region, each having a minimum of 50 patients. Consultants who look after fewer than this number and who have major clinical interests outside cystic fibrosis may not acquire the expertise needed nor warrant the expense of the essential paramedical support. Cystic fibrosis patients require individual cubicle accommodation, facilities not usually available in DGH hospitals in sufficient numbers.

Correctly priced cystic fibrosis care can provide major income for hospital trusts. However, hospital management must reinvest most of this in the service to allow the provision of optimal care and expansion to cater for the needs of a now rapidly growing population. Hospital trusts must invoice for the costs of cystic fibrosis care for each individual patient accurately and not based on an "average patient" as disease severity and the intensity of treatment can vary widely. Costs must be accurately assessed or "banded" for each patient annually for the coming financial year and the purchasers appropriately charged. Accurate billing is an essential prerequisite to proper funding for a cystic fibrosis unit.

The care system for adult patients is already in crisis in some parts of the UK. More recently established units will almost certainly encounter the above problems as their patient numbers increase. Life expectancy for adult patients will continue to rise. Those in receipt of a lung transplant still need continuing care in the cystic fibrosis centres for this multi-system disease. Severely ill patients with $\mathrm{FEV}_{1}$ values below $20 \%$ predicted can be kept alive for long periods with intensive treatments. The likely outcome of failure to increase resources is "gridlock" with existing units being incapable of accepting new referrals. Unless adequate funding is directed to adult cystic fibrosis care and further consultants appointed, patients are likely to drift to non-specialist adult respiratory units which are ill equipped for their special needs. Their alternative will be to remain with their paediatrician, usually an inadequate option for adolescents with a chronic disease, and one that the paediatric services will not be able to support in the long term. Adult patients with cystic fibrosis need powerful voices to argue their case.

Correspondence to: Dr S P Conway.

Adult Cystic Fibrosis Centre,

S P CONWAY

Seacroft Hospital,

Leeds LS14 6UH, UK

Adult Cystic Fibrosis Centre,

D E STABLEFORTH

Heartlands Hospital,

Birmingham, UK

Adult Cystic Fibrosis Centre,

Wythenshaw Hospital,

Manchester, UK

1 Corey M, Farewell V. Determinants of mortality from cystic fibrosis in Canada 1970-1989. Am F Epidemiol 1996;143:1007-17.

2 Elborn JS, Shale D, Britton JR. Cystic fibrosis: Current survival and population estimates to the year 2000. Thorax 1991;46:881-5.

3 Fredericksen B, Lanng S, Koch C, Hoiby N. Improved survival in the Danish centre-treated cystic fibrosis patient: results of aggressive treatment. Pediatr Pulmonol 1996;21:153-8.

4 Brock DJH. Prenatal screening for cystic fibrosis: 5 years' experience reviewed. Lancet 1996;347:148-50.

Clinical Standarts Advisory Group. Cystic fibrosis: access and availability of specialist services. London: HMSO, 1993.

6 Royal College of Physicians. Cystic fibrosis in adults; recommendations for care of patients in the United Kingdom. London: Royal College of care of patients

7 Cystic Fibrosis Trust. Clinical guidelines for cystic fibrosis care. London: Royal College of Physicians, 1996.

8 Robson M, Abbott J, Webb AK, Dodd M, Walsworth-Bell J. A cost description of an adult cystic fibrosis unit and cost analyses of different categories of patients. Thorax 1992;47:684-9.

9 Webb AK. Consumer, carer, provider, purchaser developing care for adults with cystic fibrosis: a specialist service. Thorax 1994;49:291-2.

10 Wildhagen MF, Verheij JBGH, Hilderink HBM, et al. Costs of care of patients with cystic fibrosis in the Netherlands in 1990-91. Thorax 1996; 51:298-301.

11 Pond MN, Conway SP. Treated pulmonary exacerbations in adults with CF do not result in loss of pulmonary function. Isr f Med Sci 1996;32: S243.

12 Conway SP. Cystic fibrosis in teenagers and young adults. Arch Dis Child 1996;75:L99-101. 